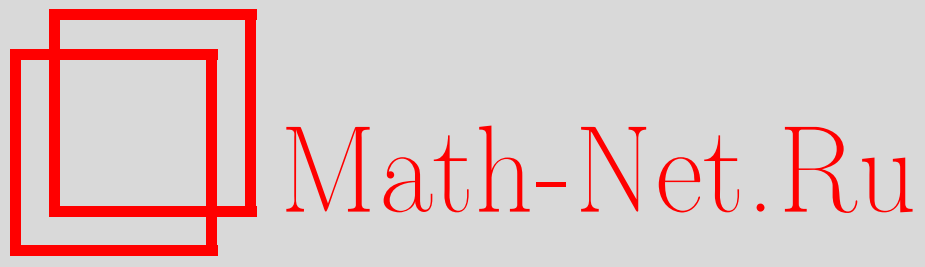

В. Д. Кошманенко, Сингулярные возмущения с бесконечной константой связи, Функи. анализ и его прил., 1999, том 33, выпуск 2, 81-84

DOI: https://doi.org/10.4213/faa358

Использование Общероссийского математического портала MathNet.Ru подразумевает, что вы прочитали и согласны с пользовательским соглашением http://www . mathnet.ru/rus/agreement

Параметры загрузки:

IP : 54.157 .27 .8

26 апреля 2023 г., 10:10:10

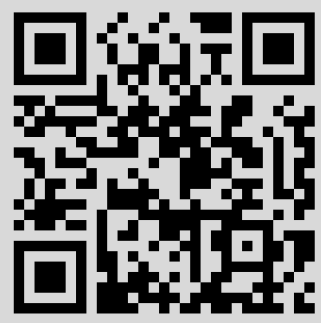


УДК 517.9

\title{
Сингулярные возмущения с бесконечной константой связи
}

\author{
(C) 1999. В. Д. Кошманенко
}

1. Пусть $A \geqslant 1$ - самосопряженный оператор в комплексном сепарабельном гильбертовом пространстве $\mathscr{H}$ с нормой $\|$ • || и скалярным произведением $(\cdot, \cdot)$. Рассмотрим часть $A$-шкалы гильбертовых пространств $\mathscr{H}_{-2} \supset \mathscr{H}_{-1} \supset \mathscr{H}_{\supset} \mathscr{H}_{1} \supset \mathscr{H}_{2}$, где пространство $\mathscr{H}_{2}$ с нормой $\|\varphi\|_{2}:=\|A \varphi\|$ совпадает с областью определения $\mathscr{D}(A)$ оператора $A, \mathscr{H}_{1}=\mathscr{D}\left(A^{1 / 2}\right)$ с нормой $\|\varphi\|_{1}:=\left\|A^{1 / 2} \varphi\right\|$, а $\mathscr{H}_{-2}, \mathscr{H}_{-1}$ обозначают сопряженные к ним пространства. Очевидно, что оператор $A$ действует унитарно из $\mathscr{H}_{2}$ в $\mathscr{H}$ и изометрично из $\mathscr{H}_{1}$ в $\mathscr{H}_{-1}$. Поэтому его замыкание $\mathbf{A}: \mathscr{H}_{1} \rightarrow \mathscr{H}_{-1}$ - унитарный оператор и справедливы равенства $(\omega, \mathbf{A} \varphi)_{-1}=\langle\omega, \varphi\rangle=\left(\mathbf{A}^{-1} \omega, \varphi\right)_{1}, \omega \in \mathscr{H}_{-1}, \varphi \in \mathscr{H}_{1}$, а также $\langle f, \varphi\rangle=(f, \varphi), f \in \mathscr{H}, \varphi \in \mathscr{H}_{1}$, где $\langle\cdot, \cdot\rangle$ - дуальное скалярное произведение между $\mathscr{H}_{1}$ и $\mathscr{H}_{-1}$ (см. [1]).

Пусть оператор $V \neq 0$ с областью определения $\mathscr{D}(V)=\mathscr{H}_{1}$ и областью значений $\mathscr{R}(V) \subset \mathscr{H}_{-1}$ ограничен и самосопряжен в том смысле, что $\langle V \varphi, \psi\rangle=$ $\langle\varphi, V \psi\rangle, \varphi, \psi \in \mathscr{H}_{1}$. Оператор $V$ называется [2] (ср. [3]) сингулярньлм воз-

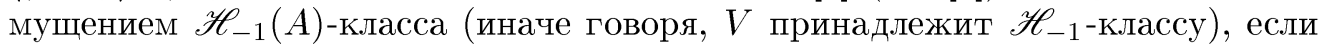
множество $\mathscr{D}:=(\operatorname{Ker} V) \cap \mathscr{D}(A)$ плотно в $\mathscr{H}$. Заметим (см. [4, Theorem A.1]), что условие $(\mathscr{R}(V))^{\mathrm{cl},-1} \cap \mathscr{H}=\{0\}\left({ }^{\mathrm{cl},-1}\right.$ означает замыкание в $\left.\mathscr{H}_{-1}\right)$ является необходимым и достаточным для того, чтобы множество $\operatorname{Ker} V$ было плотным в $\mathscr{H}$.

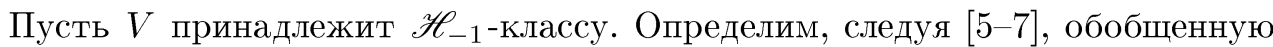
сумму $A+V$ операторов $A$ и $V$ как «сужение» обычной суммы $\mathbf{A}+V: \mathscr{H}_{1} \rightarrow$ $\mathscr{H}_{-1}$ на $\mathscr{H}$, т. е. $\mathscr{D}(A+V)=\left\{\varphi \in \mathscr{H}_{1} \mid \mathbf{A} \varphi+V \varphi \in \mathscr{H}\right\},(A+V) \varphi=\mathbf{A} \varphi+V \varphi$.

Лемма 1 [7]. Предположим, ито оператор $\left(I+\mathbf{A}^{-1} V\right)^{-1}$ существует $u$ ограничен в $\mathscr{H}_{1}$ (I - тождественное преобразование). Тогда обобщенная сумма $A+V$ является самосопряженным оператором в $\mathscr{H}$.

В частности, если $V$ положителен, $\langle V \varphi, \varphi\rangle \geqslant 0, \varphi \in \mathscr{H}_{1}$, то оператор $A_{\alpha}=A \dot{+} \alpha V$ самосопряжен в $\mathscr{H}$ для всех $\alpha>0$. При этом, очевидно, операторы $A, A_{\alpha}$ совпадают на $\mathscr{D}$ и, следовательно, являются различными самосопряженными расширениями симметрического оператора $\dot{A}=A\left|\mathscr{D}=A_{\alpha}\right| \mathscr{D}$.

Пусть $A_{\infty}$ обозначает фридрихсово расширение оператора $\dot{A}$. Отметим, что $A_{\infty} \neq A$, так как из условия, что $V \neq 0$ принадлежит $\mathscr{H}_{-1}$-классу, следует, что множество $\mathscr{D}=\mathscr{D}(\dot{A})$ плотно лишь в $\mathscr{H}$, но не в $\mathscr{H}_{1}$.

В $[8,9]$ (см. также [6]) доказано, что в случае сингулярного возмущения ранга один $V=\langle\cdot, \omega\rangle \omega, \omega \in \mathscr{H}_{-1} \backslash \mathscr{H}$, для оператора $A_{\alpha}=A \dot{+} \alpha V$ справедлив следующий факт: в сильном резольвентном смысле $\lim _{\alpha \rightarrow \infty} A_{\alpha}=A_{\infty}$. Здесь этот результат обобщается на сингулярные возмущения произвольного ранга.

ТеоремА. Пусть $V: \mathscr{H}_{1} \rightarrow \mathscr{H}_{-1}$ - сингулярное возмущение $\mathscr{H}_{-1}$ класса, причем оператор $V$ положителен, если его рана бесконечен. Предположим, что операторы $A$ и $A_{\infty}$ взаимно просты [10]. Тогда обобшенная сумма $A_{\alpha}=$ 
$A \dot{+} \alpha V, \alpha>0,-$ самосопряженньй оператор в $\mathscr{H}$ и в сильном резольвентном смысле $\lim _{\alpha \rightarrow \infty} A_{\alpha}=A_{\infty}$.

Напомним (см. $[10,11])$, что условие взаимной простоты для пары $A$ и $A_{\infty}$ означает, что симметрический оператор $\dot{A}=A\left|\mathscr{D}=A_{\infty}\right| \mathscr{D}$ является их максимальной общей частью. Это условие выполняется автоматически в случае возмущения ранга один. Отметим, что в случае, когда оператор $V$ положителен, существование сильного резольвентного предела следует из монотонного возрастания $A_{\alpha}$ при $\alpha \rightarrow \infty$ и операторного неравенства $A_{\alpha}<A_{\infty}$.

Доказательство теоремы использует частный случай (см. ниже соотношение (1)) формулы Крейна для резольвент [10-13], а также обобщенный аналог (соотношение (2)) резольвентного тождества для возмущенного оператора $[7,14]$.

2. Пусть $\mathscr{M}_{2}$ обозначает замкнутое в $\mathscr{H}_{2}=\mathscr{D}(A)$ подпространство $\mathscr{H}_{2} \cap$ $\operatorname{Ker} V$, т.е. множество $\mathscr{D}$. Оно плотно в $\mathscr{H}$, поскольку $V$ принадлежит $\mathscr{H}_{-1}$-классу. Пусть $\mathscr{M}_{1}$ обозначает замыкание подпространства $\mathscr{M}_{2}$ в $\mathscr{H}_{1}$. Заметим, что $\mathscr{M}_{2} \neq \mathscr{H}_{2}$, а также $\mathscr{M}_{1} \neq \mathscr{H}_{1}$, поскольку $V \neq 0$. Таким образом, в смысле ортогонального разложения имеем $\mathscr{H}_{2}=\mathscr{M}_{2} \oplus \mathscr{N}_{2}$ и $\mathscr{H}_{1}=\mathscr{M}_{1} \oplus \mathscr{N}_{1}$. Пусть $\mathscr{M}_{0}=\mathscr{R}(\dot{A})$ обозначает область значений симметрического оператора $\dot{A}$, а $\mathscr{N}_{0}:=\operatorname{Ker}(\dot{A})^{*}$ - его дефектное подпространство. Тогда пространство $\mathscr{H}$ также представимо в виде ортогональной суммы: $\mathscr{H}=\mathscr{M}_{0} \oplus \mathscr{N}_{0}$. Далее, так как по построению $\mathscr{M}_{2} \subset \mathscr{M}_{1}$ и $\mathscr{N}_{1} \perp \mathscr{M}_{1}$ в $\mathscr{H}_{1}$, то для любых $\eta_{1} \in \mathscr{N}_{1}$, $\mu_{2} \in \mathscr{M}_{2}$ и $\mu_{0}=A \mu_{2} \in \mathscr{M}_{0}$ имеем $\left(\eta_{1}, \mu_{0}\right)=\left(\eta_{1}, A \mu_{2}\right)=\left(\eta_{1}, \mu_{2}\right)_{1}=0$. Это означает, что $\mathscr{N}_{1} \subset \mathscr{N}_{0}$. Этот факт, а также условие взаимной простоты пары $A$ и $A_{\infty}$ гарантируют корректность определения и обратимость оператора $B$ в $\mathscr{N}_{0}$, задаваемого следующим образом. Пусть $P_{\mathscr{N}_{i}}$ обозначает ортогональный проектор на подпространство $\mathscr{N}_{i} \subset \mathscr{H}_{i}, i=-1,0,1$. Положим

$$
B \eta=A \varphi, \quad \mathscr{D}(B):=\left\{\eta \in \mathscr{N}_{1} \mid \eta=P_{\mathscr{N}_{1}} \varphi, \varphi \in \mathscr{N}_{2} \subset \mathscr{D}(A)\right\} .
$$

Лемма 2 [2]. Справедливо равенство

$$
A^{-1}=A_{\infty}^{-1}+B^{-1} P_{\mathscr{N}_{0}} .
$$

Равенство (1) - частный случай формулы Крейна для резольвент (см. [1013]). Поясним, что, поскольку оператор $A$ является одним из самосопряженных расширений симметрического оператора $\dot{A}$, для его резольвенты справедливо представление $(A-z I)^{-1}=\left(A_{\infty}-z I\right)^{-1}+B_{z}^{-1} P_{\mathscr{N}_{z}}$, где $P_{\mathscr{N}_{z}}-$ opтогональный проектор на подпространство $\mathscr{N}_{z}=\operatorname{Ker}\left(\dot{A}^{*}-\bar{z} I\right)$, а $B_{z}$ служит операторным параметром, фиксируюшим расширение оператора $A$. Нетривиальный факт (см. [2]) состоит в том, что $B_{z=0}=B$.

Из (1) получаем описание оператора $A_{\infty}: \mathscr{D}\left(A_{\infty}\right)=\left\{\psi \in \mathscr{H}_{1} \mid \psi=\varphi-\right.$ $\left.B^{-1} P_{\mathscr{N}_{0}} A \varphi=\varphi-P_{\mathscr{N}_{1}} \varphi, \varphi \in \mathscr{D}(A)\right\}, A_{\infty} \psi=A \varphi$.

Пусть $\mathbf{A}_{z}=\mathbf{A}-z I, \mathbf{A}_{\alpha, z}=\mathbf{A}+\alpha V-z I, \alpha>0, z \in \rho=\rho(A) \cap \rho\left(A_{\alpha}\right)$, где $\rho(\cdot)$ - резольвентное множество. В [7] показано, что области значений операторов $\mathbf{A}_{z}, \mathbf{A}_{\alpha, z}$ совпадают со всем пространством $\mathscr{H}_{-1}$ и, более того, обратные операторы $\mathbf{R}_{z}:=\mathbf{A}_{z}^{-1}, \mathbf{R}_{z}^{\alpha}:=\mathbf{A}_{\alpha, z}^{-1}$ - ограниченные биекции из $\mathscr{H}_{-1}$ в $\mathscr{H}_{1}$. При этом очевидно, что их сужения на $\mathscr{H}$ совпадают с резольвентами $R_{z}=$ $(A-z I)^{-1}, R_{z}^{\alpha}=\left(A_{\alpha}-z I\right)^{-1}$ операторов $A$ и $A_{\alpha}$. 
Лемма 3 [7]. На $\mathscr{H}_{-1}$ справедливо обобщенное резольвентное тождество

$$
\mathbf{R}_{z}^{\alpha}-\mathbf{R}_{z}=-\alpha \mathbf{R}_{z}^{\alpha} V \mathbf{R}_{z}=-\alpha\left(I+\alpha \mathbf{R}_{z} V\right)^{-1} \mathbf{R}_{z} V \mathbf{R}_{z}, \quad z \in \rho,
$$

где $\left(I+\alpha \mathbf{R}_{z} V\right)^{-1}: \mathscr{H}_{1} \rightarrow \mathscr{H}_{1}$ - ограниченная биекиия.

3. Приведем кратко доказательство теоремы. Из (2) следует, что на $\mathscr{H}$ выполняется обычное резольвентное тождество [14]

$$
R_{z}^{\alpha}-R_{z}=-\mathbf{R}_{z}^{\alpha} V R_{z}=-\alpha\left(I+\alpha \mathbf{R}_{z} V\right)^{-1} \mathbf{R}_{z} V R_{z} .
$$

Далее значение константы связи $\alpha$ предполагается достаточно большим и таким, что $0 \in \rho$. Это возможно, так как оператор $V$ либо положителен, либо имеет конечный ранг. Теперь из резольвентного тождества следует, что

$$
A_{\alpha}^{-1}-A^{-1}=-\alpha \mathbf{A}_{\alpha}^{-1} V A^{-1}=-\alpha\left(I+\alpha \mathbf{A}^{-1} V\right)^{-1} \mathbf{A}^{-1} V A^{-1},
$$

где $\left(I+\alpha \mathbf{A}^{-1} V\right)^{-1}: \mathscr{H}_{1} \rightarrow \mathscr{H}_{1}$ - ограниченный оператор.

Рассмотрим оператор $\mathbf{A}^{-1} V \mathbf{A}^{-1}: \mathscr{H}_{-1} \rightarrow \mathscr{H}_{1}$. Он действует нетривиально только из $\mathscr{N}_{-1}:=\mathbf{A} \mathscr{N}_{1}$ в $\mathscr{N}_{1}$, так как $\mathscr{M}_{1}=\operatorname{Ker} V$. Поэтому его сужение на $\mathscr{H}$ представимо в виде

$$
\mathbf{A}^{-1} V A^{-1}=\mathbf{A}^{-1} V A^{-1} P_{\mathscr{N}_{0}}=P_{\mathscr{N}_{1}} \mathbf{A}^{-1} P_{\mathscr{N}_{-1}} V P_{\mathscr{N}_{1}} A^{-1} P_{\mathscr{N}_{0}}=\check{\mathbf{A}}^{-1} \check{V}^{-1} P_{\mathscr{N}_{0}},
$$

где введены обозначения $\check{\mathbf{A}}=P_{\mathscr{N}_{-1}} \mathbf{A} P_{\mathscr{N}_{1}}, \check{V}=P_{\mathscr{N}_{-1}} V P_{\mathscr{N}_{1}}$ и учтено равенство нулю оператора $V A^{-1}$ на подпространстве $\mathscr{M}_{0}\left(A^{-1} \mathscr{M}_{0}=\mathscr{M}_{2} \subset \mathscr{M}_{1}=\right.$ $\operatorname{Ker} V)$, а также то, что для любого $\eta_{2} \in \mathscr{N}_{2}\left(\mathscr{N}_{2}=A^{-1} \mathscr{N}_{0}\right)$ выполняется равенство $V \eta_{2}=V P_{\mathscr{N}_{1}} \eta_{2}$. Таким образом, рассматриваемое сужение действует нетривиально только из $\mathscr{N}_{0}$ в $\mathscr{N}_{1} \subset \mathscr{N}_{0}$ и как оператор в $\mathscr{N}_{0}$ имеет вид $\check{\mathbf{A}}^{-1} \check{V} B^{-1}$, поскольку $\mathscr{D}\left(B^{-1}\right)=\mathscr{N}_{0}$. Этот оператор, очевидно, обратим, $\left(\check{\mathbf{A}}^{-1} \check{V} B^{-1}\right)^{-1}=B \check{V}^{-1} \check{\mathbf{A}}$, и действует из $\mathscr{N}_{1} \subset \mathscr{N}_{0}$ в $\mathscr{N}_{0}$. Далее, сужение оператора $\left(I+\alpha \mathbf{A}^{-1} V\right)^{-1}$ на подпространство $\mathscr{N}_{1}$ в принятых обозначениях представимо в виде $\left(I_{\mathscr{N}_{1}}+\alpha \check{\mathbf{A}}^{-1} \check{V}^{-1}\right.$, где $I_{\mathscr{N}_{1}}$ - тождественное преобразование в подпространстве $\mathscr{N}_{1}$.

Теперь, благодаря совпадению операторов $A_{\alpha}^{-1}$ и $A^{-1}$ на подпространстве $\mathscr{M}_{0}$, соотношение (3) можно переписать в виде

$$
\begin{aligned}
A_{\alpha}^{-1}-A^{-1} & =-\left(\left(\alpha \check{\mathbf{A}}{ }^{-1} \check{V} B^{-1}\right)^{-1}\left(I_{\mathscr{N}_{1}}+\alpha \check{\mathbf{A}}^{-1} \check{V}\right)\right)^{-1} P_{\mathscr{N}_{0}} \\
& =-\left(\alpha^{-1} B \check{V}^{-1} \check{\mathbf{A}}\left(I_{\mathscr{N}_{1}}+\alpha \check{\mathbf{A}}^{-1} \check{V}\right)\right)^{-1} P_{\mathscr{N}_{0}} \\
& =-\left(\alpha^{-1} B \check{V}^{-1} \check{\mathbf{A}}+B\right)^{-1} P_{\mathscr{N}_{0}} .
\end{aligned}
$$

Из последнего равенства видно, что $A_{\alpha}^{-1}-A^{-1} \rightarrow-B^{-1} P_{\mathscr{N}_{0}}$ при $\alpha \rightarrow \infty$ в сильном смысле, так как $\alpha^{-1} B \check{V}^{-1} \check{\mathbf{A}} \rightarrow 0$. Следовательно, благодаря (1), $A_{\alpha}^{-1} \rightarrow A_{\infty}^{-1}$ при $\alpha \rightarrow \infty$, что эквивалентно сильной резольвентной сходимости $A_{\alpha} \mathrm{K} A_{\infty}$.

В заключение отметим, что теорема справедлива и в случае, когда $V$ допускает разложение в сумму положительного оператора и оператора конечного ранга.

Автор признателен Ю. М. Березанскому за ряд полезных замечаний. 


\title{
ЛитеРАТУРА
}

1. Березанский Ю. М., Ус Г. Ф., ШІефтель З. Г. Функциональный анализ. Выща школа, Киев, 1990. 2. Albeverio S., Koshmanenko V. Form-sum approximations of singular perturbations of self-adjoint operators. BiBoS Preprint Nr. 771/4/97, 1997. 3. Кошманенко В. Д. Сингулярные билинейные формы в теории возмущений самосопряженных операторов. Наукова думка, Киев, 1993. 4. Albeverio S., Karwowski W., Koshmanenko V. Math. Nachr., 173, 5-24 (1995). 5. Березанский Ю. М. В кн.: Спектральный анализ дифференциальных операторов. Институт математики, Киев, 1980, с. 83-106. 6. Крейн М. Г., Яврян В. А. J. Operator Theory, 6, 155-191 (1981). 7. Albeverio S., Koshmanenko $V$. On the problem of the right Hamiltonian under singular form-sum perturbations. SFB 237 Preprint, Nr. 375, Institute für Mathematik Ruhr-Universität-Bochum, 1997. 8. Gesztesy F., Simon B. J. Funct. Anal., 128, 245-252 (1995). 9. Albeverio S., Koshmanenko $V$. Some remarks on the Gesztesy-Simon version of rank-one perturbations. BiBoS Preprint, Nr. 727/5/96, 1996. 10. Ахиезер Н. И., Глазман И. М. Теория линейных операторов в гильбертовом пространстве. М., 1966. 11. Крейн М. Г. Матем. сб., 20, № 3, 431-495 (1947). 12. Бирман М. Ш. Матем. сб., 38 (80), № 4, 431-450 (1956). 13. Alonso A., Simon B. J. Operator Theory, 4, 251-270 (1980). 14. Яфаев Д. Р. Математическая теория рассеяния. Санкт-Петербургский университет, С.-Петербург, 1994.

Институт математики НАН Украины, Киев e-mail: kosh@imath.kiev.ua

Поступило в редакцию 15 января 1998 г.

\section{Спектральные свойства разностного трехчастичного оператора Шрёдингера*}

\author{
(c) 1999. С. Н. ЛАКАев, Ж. И. АБдУЛЛАев
}

1. Введение. В спектральном анализе трехчастичного непрерывного оператора Шрёдингера имеется следующий замечательный результат: если в системе трех частиц, взаимодействующих с помощью парных короткодействующих потенциалов, ни одна из трех двухчастичных подсистем не имеет связанных состояний с отрицательной энергией, но по меньшей мере две из них имеют резонанс с энергией в нуле, то у этой трехчастичной системы существует бесконечное число трехчастичных связанных состояний с отрицательной энергией, накапливающихся к нулю. Этот эффект впервые был обнаружен Ефимовым [1]. Строгое математическое доказательство существования эффекта Ефимова было проведено впервые в работе Яфаева [2].

В моделях физики твердого тела [3], а также решетчатой теории поля [4] возникают так называемые дискретные операторы Шрёдингера, являющиеся решетчатым аналогом обычного трехчастичного оператора Шрёдингера в непрерывном пространстве.

Хотя дискретный оператор Шрёдингера ограничен и возмущение в парной задаче - компактный оператор, спектр дискретного оператора для системы трех квантовых решетчатых частиц имеет более сложный характер по сравнению с непрерывным случаем. 